PROCEEDINGS OF THE

AMERICAN MATHEMATICAL SOCIETY

Volume 100, Number 3 , July 1987

\title{
RANDOM REALS AND SOUSLIN TREES
}

\author{
RICHARD LAVER
}

\begin{abstract}
It is consistent that there are no Souslin trees in any random extension of $V$; thus, the continuum can be singular of cofinality $\omega_{1}$ with Souslin's hypothesis holding.
\end{abstract}

In [4], Roitman proved that adding a Cohen real causes $\mathrm{MA}_{\aleph_{1}}$ to fail: If $g$ is a Cohen real over $V$, then in $V[g]$ there is a ccc partial ordering $P$ such that $P \times P$ does not have the ccc (see also Galvin [3], where the existence of such a $P$ is derived from $\mathrm{CH}$ ). Shelah [5] improved Roitman's result to: If $g$ is a Cohen real over $V$, then in $V[g]$ there is a Souslin tree. Kunen (see [4]) noted that Roitman's theorem also holds for random extensions: If $r$ is random over $V$, then a ccc $P$, with $P \times P$ not ccc, exists in $V[r]$. Left open was the random real analogue of Shelah's theorem, that is, whether it is a theorem of ZFC that adding a random real adds a Souslin tree.

The question whether adding a Sacks real adds a Souslin tree is independent of ZFC+SH. Namely, suppose $s$ is a Sacks real over $V$. Carlson showed (unpublished) that if $V$ satisfies a fragment of axiom $\mathrm{A}+2^{\aleph_{0}}>\aleph_{1}$ (which is consistent relative to the consistency of ZFC), then $V[s]$ satisfies $\mathrm{MA}_{\aleph_{1}}$, and the author (unpublished) showed that if $V$ satisfies $\mathrm{CH}$, then $V[s]$ satisfies $\diamond_{\omega_{1}}$.

The result of this paper is that if $\mathrm{MA}_{\aleph_{1}}$ holds, then the adjunction of any number of random reals does not add a Souslin tree. Recall that if $\mathrm{MA}_{\kappa}$ holds, then any tree $T$ of size $\leq \kappa$ with no paths of length $\omega_{1}$ is special, that is, $T$ is the union of countably many antichains (Baumgartner, Malitz, and Reinhardt [1]).

THEOREM. If $\mathrm{MA}_{\kappa}$ holds and $B$ is a measure algebra, then in $V^{B}$ every tree of size $\leq \kappa$ with no paths of length $\omega_{1}$ is special.

This theorem fills in the last part to the result that the continuum can be arbitrary with Souslin's hypothesis holding. That Souslin's hypothesis can hold with the continuum being an arbitrary regular cardinal, or an arbitrary singular cardinal of cofinality greater than $\omega_{1}$, is the work of Solovay and Tennenbaum [7] and Jensen (see [2]). To get that $2^{\aleph_{0}}$ can be any singular cardinal of cofinality $\omega_{1}$ with the Souslin hypothesis holding, begin with a model of $\mathrm{MA}_{\aleph_{1}}$ and add $\lambda$ random reals ( $\lambda$ any cardinal satisfying $\mathrm{cf} \lambda=\omega_{1}$ and $\lambda^{\aleph_{0}}=\lambda$ ).

Also, by starting with a model of $\mathrm{MA}_{\aleph_{1}}$ and adding at least measurably many random reals, the consistency that there is a real valued measurable cardinal $\leq$ $2^{\aleph_{0}}$ (see Solovay [6]) and Souslin's hypothesis holds, is obtained, relative to the consistency of a measurable cardinal.

Proof of THE TheOREM. Assume $\mathrm{MA}_{\kappa}$. Suppose $B$ is an atomless measure algebra via a countably additive measure $\mu: B \rightarrow[0,1]$. Suppose $T$ is a term in

Received by the editors April 14, 1986.

1980 Mathematics Subject Classification (1985 Revision). Primary 03E35. 
the forcing language of $B$ for a tree of the form $\left(\kappa,<_{T}\right)$, and suppose $T$ is forced to have no paths of length $\omega_{1}$. A $B$-term $F$ will be constructed such that it is forced that $F$ is a partition of $T$ into $\aleph_{0}$-many antichains. The first two lemmas show that the poset, used to produce $F$ by an application of Martin's axiom, has the ccc. Fix a uniform ultrafilter $\mathcal{U}$ on $\omega_{1}$.

Lemma 1. Suppose $0<\gamma<\varepsilon, X \in \mathcal{U}, b_{\beta} \in B(\beta \in X)$, and each $\mu\left(b_{\beta}\right) \geq \varepsilon$. Then for some $c \in B, \mu(c)>0$, and for all $c^{\prime} \leq c,\left\{\beta: \mu\left(c^{\prime} \wedge b_{\beta}\right) \geq \gamma \cdot \mu\left(c^{\prime}\right)\right\} \in \mathcal{U}$.

PROOF. If for every $c$ there is a counterexample $c^{\prime} \leq c$, take a partition of 1 into such counterexamples. Let $C$ be a finite subset of the partition such that $\mu(\bigvee C) \geq 1-(\varepsilon-\gamma)$. For each $c^{\prime} \in C, X_{c^{\prime}}=\left\{\beta: \mu\left(c^{\prime} \wedge b_{\beta}\right)<\gamma \cdot \mu\left(c^{\prime}\right)\right\} \in \mathcal{U}$. Pick $\beta \in \bigcap_{c^{\prime} \in C} X_{c^{\prime}}$. Then $\mu\left(b_{\beta}\right)<\gamma \cdot \mu(\bigvee C)+(\varepsilon-\gamma) \leq \varepsilon$, a contradiction.

LEMMA 2. Suppose $\varepsilon>0, X \in\left[\omega_{1}\right]^{\aleph_{1}}, X_{\alpha} \in \mathcal{U}(\alpha \in X)$, and $\mu\left(b_{\alpha \beta}\right) \geq \varepsilon$ $\left(\alpha \in X, \beta \in X_{\alpha}\right)$. Define, in $V\left[G_{B}\right], R(\alpha, \bar{\alpha}) \Leftrightarrow$ for some $\beta, b_{\alpha \beta} \wedge b_{\bar{\alpha} \beta} \in G_{B}$. Then for some $c>0$ in $B$,

$$
c \Vdash \exists Y \in[X]^{\aleph_{1}} \forall \alpha, \bar{\alpha} \in Y R(\alpha, \bar{\alpha}) .
$$

ProOF. Pick $\gamma<\varepsilon$. For each $\alpha \in X$ apply Lemma 1 to the sequence $b_{\alpha \beta}$ $\left(\beta \in X_{\alpha}\right)$, getting a $c_{\alpha} \in B$. Then by the ccc-ness of $B$ there is a $c \in B$ forcing that $W=\left\{\alpha: c_{\alpha} \in G_{B}\right\}$ has size $\aleph_{1}$.

We claim that $c$ works. Namely pick $N$ so that $N \cdot \gamma>1$. Using $\aleph_{1} \rightarrow\left(\aleph_{1}, N\right)^{2}$ in $V\left[G_{B}\right]$, either there is a $Y$ as in the lemma or there is an $e \in B, e \leq c$, and $\alpha_{0}<\alpha_{1}<\cdots<\alpha_{N-1}$ such that $e \Vdash$ each $\alpha_{i} \in W$ and $\neg R\left(\alpha_{i}, \alpha_{j}\right)(i<j \leq N-1)$. Suppose such an $e$ exists. Then $e \leq c_{\alpha_{0}} \wedge \cdots \wedge c_{\alpha_{N-1}}$, so by the choice of the $c_{\alpha_{i}}$ 's there are $X_{\alpha_{i}} \in \mathcal{U}$ such that for $\beta \in X_{\alpha_{i}}, \mu\left(b_{\alpha_{i} \beta} \wedge e\right) \geq \gamma \cdot e$. Pick $\beta \in \bigcap_{i<N} X_{\alpha_{i}}$. Since $N \cdot \gamma>1$, $e \wedge b_{\alpha_{i} \beta} \wedge b_{\alpha_{j} \beta}>0$ for some $i<j<N$. But $e \wedge b_{\alpha_{i} \beta} \wedge b_{\alpha_{j} \beta}$ forces $R\left(\alpha_{i}, \alpha_{j}\right)$, a contradiction.

A poset $P$ for an application of $\mathrm{MA}_{\kappa}$ will be constructed. For $\alpha<\kappa$, let $A_{\alpha}$ be the countable set $\left\{\delta:\left[\mid \delta<_{T} \alpha \|>0\right\}\right.$.

Define complete subalgebras $B_{\alpha}$ of $B$ by induction on $\alpha<\kappa$. $B_{0}$ is an arbitrary countably generated atomless subalgebra of $B$. For $0<\alpha<\kappa$, let $B_{\alpha}$ be the complete subalgebra of $B$ generated by

$$
\bigcup_{\delta \in A_{\alpha}}\left(B_{\delta} \cup\left\{\left[\mid \delta<_{T} \alpha \|\right]\right\}\right)
$$

Then each $B_{\alpha}$ is isomorphic to the countably generated atomless measure algebra. Fix a countable dense $C_{\alpha} \subseteq B_{\alpha}-\{0\}$.

A condition in $P$ is a function $f$ with domain of the form $E \times W$, where $E \in[\kappa]<\aleph_{0}$ and $W \in[\omega]<\kappa_{0}$, such that

(1) $f(\alpha, n) \in B_{\alpha}$, and either $f(\alpha, n)=0$ or $\mu(f(\alpha, n))>\frac{1}{2}$.

(2) $f(\alpha, n) \wedge f(\beta, n) \Vdash \alpha$ and $\beta$ are $T$-incomparable. (If $f(\alpha, n) \wedge f(\beta, n)=0$, we say that this forcing relation holds vacuously.)

For $f, g \in P, g$ extends $f$ if $E_{f} \subseteq E_{g}$ and $W_{f} \subseteq W_{g}$, and for each $(\alpha, n) \in$ $\operatorname{dom} f, f(\alpha, n) \geq g(\alpha, n)$ and if $f(\alpha, n)>0$, then $g(\alpha, n)>0$. Thus $P$ adds a sequence $d_{\alpha, n}(\alpha<\kappa, n<\omega)$ where $d_{\alpha, n} \in B$ is either 0 or is of measure $\frac{1}{2}$ and obtained by amoeba forcing on $B_{\alpha}$.

Let $f \sim g$ denote that $f$ and $g$ are compatible. If $n \in W_{f}$ let $f \uparrow n=f \uparrow E_{f} \times\{n\}$. Evidently, if $W_{f}=W_{g}$ and $f \uparrow n \sim g \uparrow n$, for each $n \in W_{f}$, then $f \sim g$. 
LEMMA 3. P has the ccc.

ProOF. Suppose $f_{\gamma} \in P\left(\gamma<\omega_{1}\right)$ and $f_{\gamma} \not f f_{\gamma^{\prime}}\left(\gamma \neq \gamma^{\prime}\right)$. We may assume that all the $W_{\gamma}$ 's are the same set $W$, and by the $\Delta$-system lemma we may assume that all the $E_{\gamma}$ 's have size $n$, and that for some $E$ and all $\gamma \neq \gamma^{\prime}, E_{\gamma} \cap E_{\gamma^{\prime}}=E$. We may further assume that for some $\varepsilon>0$ and all $\gamma$, if $\mu\left(f_{\gamma}(\langle\alpha, n\rangle)\right)>\frac{1}{2}$, then $\mu\left(f_{\gamma}(\langle\alpha, n\rangle)\right) \geq \frac{1}{2}+\varepsilon$. For $\alpha \in E$, approximate each $f_{\gamma}(\langle\alpha, n\rangle)$ by finite Boolean combinations of members of $C_{\alpha}$ to within accuracy $\varepsilon / 3$. We may assume that these approximations are independent of $\gamma$, and thus that $f_{\gamma} \uparrow E \times W \sim F_{\gamma^{\prime}} \uparrow E \times W$. So we may assume $E=\varnothing$.

Since $\operatorname{Card}\left\{\beta:\left[\left\|\beta<_{T} \alpha\right\|\right]>0\right\} \leq \aleph_{0}$ for each $\alpha<\kappa$, we may assume that if $\gamma<\gamma^{\prime}, \beta \in E_{\gamma^{\prime}}$, and $\alpha \in E_{\gamma}$, then $\left[\mid \beta<_{T} \alpha \|=0\right.$. If $f_{\gamma} \not f_{\gamma^{\prime}}$, then for some $k$, $f_{\gamma} \uparrow E_{\gamma} \times\{k\} \not f_{\gamma^{\prime}} \uparrow E_{\gamma^{\prime}} \times\{k\}$. Pick a $k$ such that

$$
\left\{\gamma:\left\{\gamma^{\prime}: f_{\gamma} \uparrow E_{\gamma} \times\{k\} \not f_{\gamma^{\prime}} \uparrow E_{\gamma} \times\{k\}\right\} \in \mathcal{U}\right\} \in \mathcal{U} .
$$

Let $\bar{f}_{\gamma}=f_{\gamma} \uparrow E_{\gamma} \times\{k\}$, and write $E_{\gamma}=\left\{\sigma_{\gamma, 0}, \sigma_{\gamma, 1}, \ldots, \sigma_{\gamma, n-1}\right\}$.

Given $\gamma<\gamma^{\prime}$ with $\bar{f}_{\gamma} \not \bar{f}_{\gamma^{\prime}}$, there must be $i, j<n$ with $\mu\left[\mid \sigma_{\gamma, i}<_{T} \sigma_{\gamma^{\prime}, j} \| \geq\right.$ $\varepsilon / n+1$. Namely, suppose for all $i, j$ that $\mu\left[\mid \sigma_{\gamma, i}<_{T} \sigma_{\gamma^{\prime}, j} \|<\varepsilon / n+1\right.$. Then $\bar{f}_{\gamma}$ and $\bar{f}_{\gamma^{\prime}}$ would be extended by $\bar{f}$, where $\operatorname{dom} f=\left(E_{\gamma} \cup E_{\gamma^{\prime}}\right) \times\{k\}, \bar{f} \uparrow E_{\gamma} \times\{k\}=\bar{f}_{\gamma}$, and for $j<n$,

$$
\bar{f}\left(\left\langle\sigma_{\gamma^{\prime}, j}, k\right\rangle\right)=\bar{f}_{\gamma^{\prime}}\left(\left\langle\sigma_{\gamma^{\prime}, j}, k\right\rangle\right)-\bigvee_{i<n}\left[\left|\dot{\sigma}_{\gamma, i}<_{T} \sigma_{\gamma^{\prime}, j}\right|\right]
$$

(this is an element of $B_{\sigma_{\gamma^{\prime}, j}}$ ).

Thus there are $i, j<n$ such that $\left\{\gamma:\left\{\gamma^{\prime}: \mu\left(b_{\gamma \gamma^{\prime}}\right) \geq \varepsilon / n+1\right\} \in \mathcal{U}\right\} \in \mathcal{U}$, where $b_{\gamma \gamma^{\prime}}=\left[\begin{array}{lll}\mid \sigma_{\gamma, i}<_{T} & \sigma_{\gamma^{\prime}, j} \|\end{array}\right]$. Apply Lemma 2 to the $b_{\gamma \gamma^{\prime}}$ 's, getting a generic extension $V\left[G_{B}\right]$ in which for some $X \in\left[\omega_{1}\right]^{\aleph_{1}}$, for every $\gamma, \bar{\gamma}$ in $X$, there is a $\gamma^{\prime}$ with $b_{\gamma \gamma^{\prime}} \wedge b_{\bar{\gamma} \gamma^{\prime}} \in G_{B}$.

In this extension, if $\gamma, \gamma^{\prime} \in X$ and $\gamma<\gamma^{\prime}$, then $\sigma_{\gamma, i}<_{T} \sigma_{\gamma^{\prime}, j}$ since $T$ is a tree. This contradiction completes the proof of Lemma 3 .

Recall that $C_{\alpha}$ is a countable dense subset of $B_{\alpha}-\{0\}$. For $\alpha<\kappa, c \in C_{\alpha}$, let $D_{\alpha, c}=\left\{f \in \mathcal{P}: \exists n\langle\alpha, n\rangle \in \operatorname{dom} f, \mu(f(\langle\alpha, n\rangle))>\frac{1}{2}, \mu(f(\langle\alpha, n\rangle)-c)<\frac{1}{2}-\frac{1}{2} \mu(c)\right\}$. Since $B_{\alpha}$ is atomless, each $D_{\alpha, c}$ is cofinal in $\mathcal{P}$. Let $\mathcal{G}$ be $\mathcal{P}$-generic over the $D_{\alpha, c}$ 's.

Let $d_{\alpha, n}=\bigwedge\left\{f(\langle\alpha, n\rangle:\langle\alpha, n\rangle \in \operatorname{dom} f, f \in \mathcal{G}\}\right.$. In $V\left[G_{B}\right]$ let $A_{n}=\left\{\alpha: d_{\alpha n} \in\right.$ $\left.G_{B}\right\}$. We claim that it is forced in $B$ that the $A_{n}$ 's are $T$-antichains and $\bigcup_{n<\omega} A_{n}=$ $\kappa$. They are antichains by the definition of $P$. Now suppose $b \Vdash \alpha \notin \bigcup_{n<\omega} A_{n}$. Since each $d_{\alpha n} \in B_{\alpha}$, we may as well assume $b \in B_{\alpha}$. Pick $c \in C_{\alpha}$ with $c-b<\frac{1}{4} \mu(c)$. Pick $f \in \mathcal{G}$ and $n<\omega$ with $\mu(f(\langle\alpha, n\rangle))>\frac{1}{2}$ and $\mu(f(\langle\alpha, n\rangle)-c)<\frac{1}{2}$. Then $b \wedge d_{\alpha n}>0$ and forces that $\alpha \in A_{n}$. This completes the claim and the theorem.

\section{REFERENCES}

1. J. Baumgartner, J. Malitz, and W. Reinhardt, Embedding trees in the rationals, Proc. Nat. Acad. Sci. U.S.A. 67 (1970), 1748-1755.

2. K. Devlin and H. Johnsbraten, The Souslin problem, Lecture Notes in Math., Vol. 406, Springer-Verlag, Berlin and New York, 1974.

3. F. Galvin, Chain conditions and products, Fund. Math. 108 (1980), 33-48.

4. J. Roitman, Adding a random or a Cohen real: topological consequences and the effects on Martin's axiom, Fund. Math. 103 (1979), 47-60. 
5. S. Shelah, Can you take Solovay's inaccessible away?, Israel J. Math. 48 (1984), 1-47.

6. R. Solovay, Real valued measurable cardinals, Proc. Sympos. Pure Math., vol. 13, Part 1, Amer. Math. Soc., Providence, R.I., 1971, pp. 397-428.

7. R. Solovay and S. Tennenbaum, Iterated Cohen extensions and Souslin's problem, Ann. of Math. 94 (1971), 201-245.

Department of Mathematics, University of Colorado, Boulder, Colorado 80309 